\title{
Coordinated Robust Routing by Dual Cluster Heads in Layered Wireless Sensor Networks
}

\author{
Mei Yang ${ }^{\dagger}$, Jianping Wang ${ }^{\ddagger}$, Zhenguo Gao*, Yingtao Jiang ${ }^{\dagger}$, Yoohwan Kim \\ ${ }^{\dagger}$ Department of Electrical and Computer Engineering, University of Nevada Las Vegas, NV 89154 \\ ¥ Department of Computer Science, Georgia Southern University, Statesboro, GA 30460 \\ School of Computer Science, Harbin Institute of Technology, Harbin, China, \\ * School of Computer Science, University of Nevada Las Vegas, NV 89154 \\ E-mail: ${ }^{\dagger}\left\{\right.$ meiyang,yingtao\}@egr.unlv.edu, ${ }^{*}$ gag@ ftcl.hit.edu.cn, ${ }^{\dagger}$ jpwang@ georgiasouthern.edu, ${ }^{\star}$ yoohwan@ $c s . u n l v . e d u$
}

\begin{abstract}
In this paper, we propose the coordinated robust routing (CRR) scheme to address the fault tolerance requirements in the layered wireless sensor networks. In the proposed scheme, the robust routing trees are constructed coordinately from the most outward layer all the way to the sink node. The coordination is achieved by selecting two dedicated cluster heads for every two clusters in one layer. The problem of selecting dual cluster heads is formulated as a transportation problem, which can be solved using network flow algorithms. By having two cluster heads for every cluster pair, the CRR scheme helps to achieve fault tolerance and energy efficiency with low degree of network redundancy as well as low inter-packet delay. The performance of the CRR scheme in terms of delay, energy consumption, and network lifetime has been confirmed through simulations and compared with the single cluster head scheme.
\end{abstract}

Keyword: Wireless sensor networks, fault tolerance, energy efficiency, cluster, robust routing.

\section{Introduction}

Recent advances in wireless communications and electronics have enabled the development of low-cost, lowpower, small-size, and multi-functional sensor nodes. These tiny sensor nodes, which consist of sensing, data processing, and communicating components, have led to the emergence and deployment of wireless sensor networks. A typical wireless sensor network consists of one or more base stations (sinks) and a large number of sensor nodes scattered in a sensor field [2]. Each of these scattered sensor nodes is capable to collect data and relay data back to the sink through a multi-hop architecture. The sink may communicate with the task manager node via the Internet or a satellite. The low-cost, rapid deployment, ability of self- organization and cooperative data-processing have made the wireless sensor networks a practical solution for a wide range of application areas, including military, industry and commercial, environment, health and home $[2,3]$.

The design of wireless sensor networks is influenced by the following major factors. 1) Energy efficiency: The most significant challenge in sensor networks is to overcome the energy constraint since each sensor node has limited power $(<0.5 \mathrm{Ah}, 1.2 \mathrm{~V}>)$, and it is hard to replenish the power in some hazardous or hostile application scenarios. 2) Fault tolerance: Some sensor nodes may fail or be blocked due to lack of power (energy) or physical damage or environmental interference. It is particularly important to provide fault tolerance in sensor networks under extreme and hostile environments, such as battle fields and wildlife fields. Such a failure of sensor nodes should not affect the overall task operation of the sensor network. 3) Scalability: The number of sensor nodes deployed in studying a phenomenon may be in the order of hundreds or thousands, or even millions for some specific applications. Apparently, seamless connectivity of newly added sensor nodes to the existing infrastructures is desirable.

For large-scale sensor networks, two different network architectures are generally considered: clustered architecture [6] and multi-hop layered architecture [5]. In the clustered architecture, sensor nodes which are close to each other are grouped into a cluster [6]. The nodes in the cluster send their data to a local cluster head, which is responsible for sending these data to the sink node. In the layered architecture, nodes that have the same hop count to the sink are partitioned into one layer [5]. The number of layers and the number of nodes in each layer are determined by the geographical distribution of the nodes and the sink location. Nodes in the same layer select one node in its adjacent layer closer to the sink as the forwarding node (cluster head). Fig. 1 shows an example of layered wireless sensor networks. 
Node 1 is the cluster head for node set $\{6,7, \cdots, 10\}$.

The election (selection) process of the cluster head in the clustered architecture is different from that in the layered architecture. In a clustered architecture, there is no guarantee that the cluster head is physically closer to the sink. It may take more energy to carry the aggregated data from the cluster head to the sink. However, in the layered architecture, the cluster head is guaranteed to be closer to the sink and it will relay the data to the sink with less energy consumption.

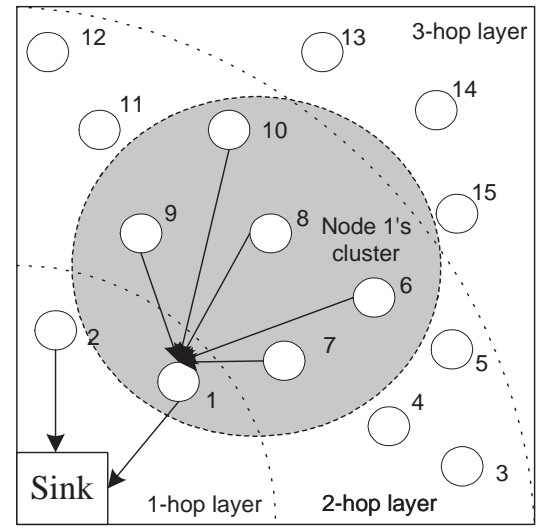

Figure 1. An example of layered wireless sensor networks.

In this paper, we consider the sensor networks based on the layered architecture. In particular, we attempt to provide fault tolerance and achieve fast data relay in the context of energy efficiency. We propose the coordinated robust routing scheme that can select two cluster heads for each cluster. The rest of the paper are organized as follows. Section 2 presents the layered architecture and its related network organization protocol and channel allocation scheme. Section 3 describes the CRR scheme. Section 4 discusses the formulation of the dual cluster heads selection problem. Section 5 presents the simulation results. Section 6 summarizes the paper.

\section{The Layered Architecture}

The layered architecture we consider in this paper is based on the the Multi-Hop Infrastructure Network Architecture (MINA) [5]. In MINA, sensor nodes are organized into layers such that the nodes having the same hop-count to the sink node belong to the same layer. The sink node is assumed to have a long transmission range to cover the whole network. Hence it can use a single broadcast transmission to reach all the nodes in the network.

Depending on their hop-count to the sink node, the neighbors of a node are classified as either inward, outward, or peer to the node [5]. Let $C_{i}$ denote the number of hops from node $i$ to the sink node. A node $j$ is defined as inward to node $i$ if $C_{j}<C_{i}$; as peer to node $i$ if $C_{j}=C_{i}$; as outward to node $i$ if $C_{j}>C_{i}$. For example, in Fig. 1, for node 7 , its inward node set, peer node set, and outward node set are $\{1,2\},\{3,4,5,6,8, \cdots, 12\},\{13,14,15\}$, respectively.

Four kinds of packets are transmitted in the layered sensor network. 1) Control packets $(C P)$ which contain the timing information for the sensor nodes to synchronize their clocks, time slot order in which the nodes should transmit their beacon packets, and the channel allocation information. 2) Cluster control packets (CCP) which contain the cluster ID, the cluster head ID, the channel allocated to the cluster, the synchronization information, and the time slot order in which the nodes should transmit their beacon packets inside a cluster. 3) Beacon packets $(B P)$ which contain the sensor node ID, the cluster ID, the cluster head ID, state, residual energy, receiver channel, buffer status, etc. 4) Data packets $(D P)$ which contain the data collected by the senor nodes.

\subsection{Network Organization Protocol}

The sensor network operates in two major phases: network discovery phase during which nodes discover their neighbors and their parameters (such as hop count, residual energy, receiver channel, buffer status, etc.), and data transmission phase during which nodes transmit and receive data (to be forwarded to the sink).

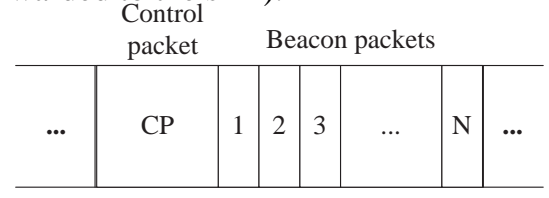

(a)

\begin{tabular}{l|c|c|c|c|c|c|c|c|c} 
Control & \multicolumn{1}{c}{$\begin{array}{c}\text { Cluster control } \\
\text { packet }\end{array}$} \\
& & $\mathrm{CCP}$ & & $\mathrm{CCP}$ & $\mathrm{DP}$ & $\mathrm{DP}$ & & $\mathrm{DP}$ & \\
\hline & $\mathrm{CP}$ & $\begin{array}{c}\text { packets } \\
1\end{array}$ & $\ldots$ & $\mathrm{H}$ & 1 & 2 & $\ldots$ & $\mathrm{P}$ & $\ldots$ \\
\hline
\end{tabular}

(b)

Figure 2. a) Network discovery super-frame. b) Data transmission super-frame.

Two kinds of super-frames, network discovery superframe and data transmission super-frame (as shown in Fig. 2 ), are used in these two phases, respectively. The network discovery super-frame consists of one $\mathrm{CP}$ and $N \mathrm{BPs}$, where $N$ is the number of nodes in the network. BPs are transmitted according to the time slot order specified in the $\mathrm{CP}$. The data transmission super-frame consists of one $\mathrm{CP}$, $H$ CCPs, and $P$ DPs, where $H$ is the maximum number of cluster heads in a cluster, and $P$ is the maximum number of nodes in a cluster. Data aggregation is performed at the end of each data transmission super-frame. The cluster head can only start to receive data packets in the next 
super-frame after finishing the data aggregation for the current super-frame. The super-frame needs to accommodate the time needed for data aggregation. The steps involved in each phase are described as follows.

In the network discovery phase, the following two steps are repeated until the most outward layer is discovered.

Step 1: The sink node broadcasts a CP to all the nodes in the network.

Step 2: After receiving the $\mathrm{CP}$, each sensor node sends a $\mathrm{BP}$ according to the order specified in the CP.

After the first round of BP exchange, the list of 1-hop layer nodes shall be made known to the sink node. These 1-hop layer nodes will update their hop count as 1. During the second round, the 2-hop layer nodes will be discovered. Each node receiving more than one BPs from 1-hop layer nodes will decide one to be its cluster head according to its residual energy (or other related parameters) and update the relevant fields in its BP. This process repeats until all layers are discovered. In the end, every sensor node knows its hop count, its cluster head and the related parameters, and its allocated time slot.

Once the network is discovered, the data transmission phase starts. The following steps are repeated in this phase.

Step 1: The sink node broadcasts a CP to all the nodes in the network. The CP now contains the channel allocation information for each cluster.

Step 2: After receiving the $\mathrm{CP}$, each cluster head sends out a CCP which contains the channel allocated, the synchronization information, and the time slot order that the nodes inside the cluster need to follow.

Step 3: After receiving the CCP, each sensor node sends the data using the allocated channel in its dedicated time slot.

\subsection{Channel Allocation}

The range of a cluster is decided by the optimal transmission range, which is relatively small [4]. Synchronization inside each cluster is easier than that among the clusters, although bandwidth requirement for inter-cluster communication is higher than that for intra-cluster coordination. Based on this observation, we propose a hybrid timedivision multiple access (TDMA)/frequency-division multiple access (FDMA)-based channel allocation scheme. Inside each cluster, TDMA is employed to take advantage of its small transmit on time [9]. Among clusters, FDMA is employed as it does not require synchronization and consumes less energy when the receiver consumes more power (as cluster heads must keep their receivers on to receive data from outward clusters) [9].
Each data packet occupies a fixed number of slots. A simple scheduling algorithm can be used to determine the time slot order inside a cluster. Channel assignment to clusters requires that adjacent clusters (either in the same layer or different layers) use different frequencies. This problem can be modelled as a graph coloring problem on the graph consisting of nodes of clusters and edges connecting adjacent clusters [5]. The sink can run the heuristic algorithm proposed in [5] to determine the channel used at each cluster.

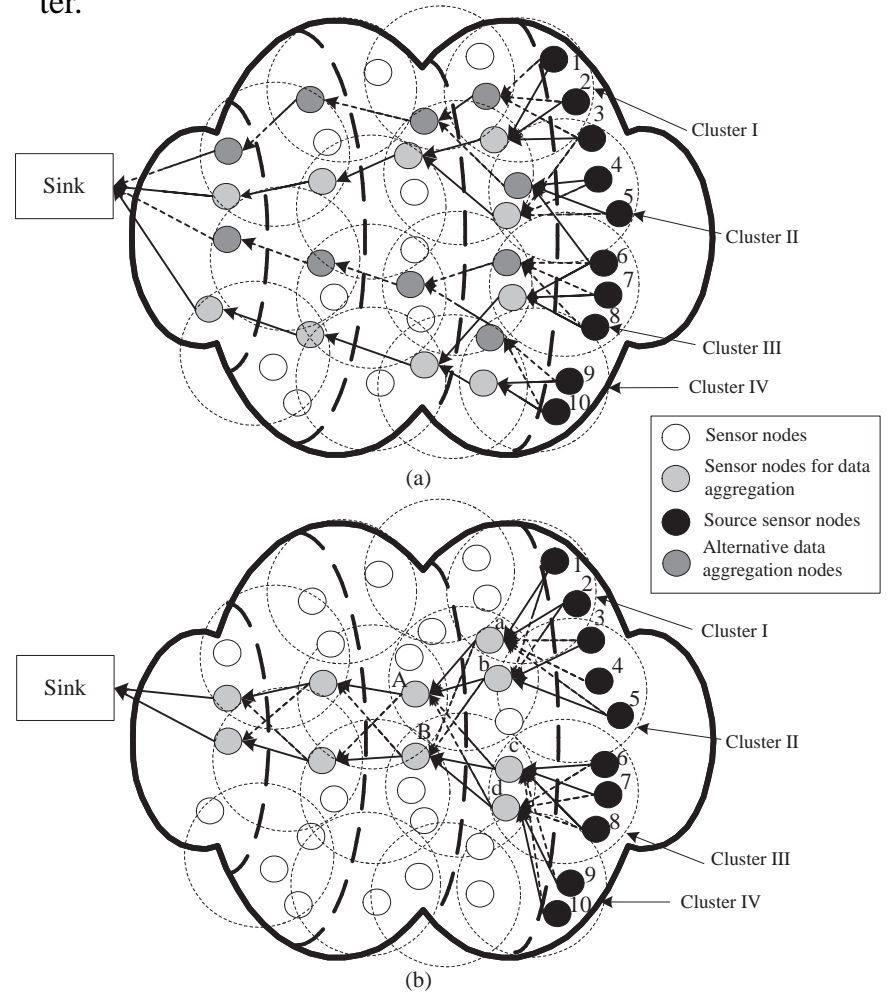

Figure 3. a) Construction of independent robust routing trees. b) Construction of coordinated robust routing trees.

\section{The Coordinated Robust Routing Scheme}

In the layered architecture, each cluster has only one inward node as its cluster head. If the inward cluster head node fails, the data from the cluster will be lost until a new inward cluster head is selected/elected. On the other hand, considering the data aggregation, the inward node cannot accept the second round data transmitted from the outward nodes until it finishes the data aggregation and forwards the data to the next hop [7], which might result in high interpacket delay.

To provide fault tolerance to the sensor networks, we need to find multiple disjoint paths [11] from each source sensor to the sink, and this process is often called robust routing. Traditionally, multiple disjoint trees, each rooted 
at a one-hop neighbor of the sink, are built [10]. Each tree will be forced to grow outward from the sink by successive branching. An example is given in Fig. 3(a), where two routing trees are constructed, and two disjoint paths exist from each source sensor node to the sink. There are 20 nodes involved in relaying data from the source nodes in the most outward layer to the sink node. These nodes have to keep powered on during the time of data transmission, which cause high energy consumption. The degree of network redundancy in this scheme is large and the data aggregation property has not been fully exploited.

In light of the idea of dual-homing [11], we propose an alternative solution, named as Coordinated Robust Routing (CRR), in which the robust routing trees from the most outward layer to the sink are constructed in a coordinated fashion. The coordination is achieved by the following way. Each pair of clusters in one layer share two dedicated cluster heads in their adjacent inward layer, one as the primary cluster head and the other as the alternative cluster head. We assume that each potential cluster head can tune to different channels to receive the data during different data transmission super-frames. For instance, clusters I and II in Fig. 3(b) share two cluster heads $a$ and $b$. In all odd super-frames, cluster head $a$ receives data from cluster I on channel $f_{1}$, and cluster head $b$ receives data from cluster II on channel $f_{2}$. In all even super-frames, cluster head $a$ tunes its receiver channel to $f_{2}$ and receives the data from cluster II; cluster head $b$ tunes its receiver channel to $f_{1}$ and receives the data from cluster I.

Similarly, clusters III and IV share the same cluster heads $c$ and $d$. In their adjacent inward layer, the cluster that nodes $a$ and $b$ belong to and the cluster that nodes $c$ and $d$ belong to share the two cluster heads $A$ and $B$ in their inward layer. Following the same way, a coordinated robust routing tree is constructed and the multiple disjoint paths from each source sensor to the sink can be readily discovered. As shown in Fig. 3(b), only 10 nodes are needed for relaying data in the CRR scheme, which is much less than that in the independent robust routing scheme (20 nodes in Fig. 3(a)).

In the CRR scheme, in case one cluster head fails, the data will be sent through the alternative cluster head in the following data transmission super-frame. In this way, the data loss is avoided and the recovery time is short. More noticeably, the network redundancy is minimized by selecting the alternative cluster head in a coordinated way. Therefore, energy efficiency is achieved.

The two cluster heads alternate to receive and aggregate data for the clusters they are assigned to. Using two separate sets of buffers to store data received from the two clusters respectively, each cluster head can process data in one buffer and simultaneously store the received data to another buffer. To achieve a shorter inter-packet delay, we can reduce the size of the super-frame by reducing the number of data packets in a super-frame. Fig. 4 shows the shorted data transmission super-frame. For example, in odd superframes, half of the nodes in the cluster send data to one of the cluster head; in even super-frames, the other half of nodes send data to the other cluster head. By such a way, the inter-packet delay is reduced by half. The cost of having two sets of buffers for each sensor node is small.

\begin{tabular}{c|c|c|c|c|c|c|c|c|c}
\multicolumn{1}{c}{$\begin{array}{c}\text { Control } \\
\text { packet }\end{array}$} \\
\begin{tabular}{c|c|c|c|c|c|c} 
Cluster control \\
packets
\end{tabular} \\
\hline & & $\mathrm{CCP}$ & & $\mathrm{CCP}$ & $\mathrm{DP}$ & $\mathrm{DP}$ & & $\mathrm{DP}$ & \\
$\ldots$ & $\mathrm{CP}$ & 1 & $\ldots$ & $\mathrm{H}$ & 1 & 2 & $\ldots$ & $\mathrm{P} / 2$ & $\ldots$ \\
& & & & & & & & & \\
\hline
\end{tabular}

\section{Figure 4. The shortened data transmission super-frame.}

\section{The Problem of Selecting Dual Cluster Heads for Cluster Pairs}

Given the pairs of clusters and the potential cluster heads they can have in the adjacent inward layer, we need to select two cluster heads for each pair of clusters such that the total remaining energy is maximally preserved in the sensor network. In a multi-hop layered architecture, such a decision might be made in each hop. Without loss of generality, we present how to select two cluster heads for each pair of clusters for the nodes in the most outward layer.

Suppose in a layered sensor network, there are $K$ layers. Layer $K$ is the most outward layer and layer 1 is the most inward layer. In layer $K-1$, the candidate cluster heads for layer $K$ are given in set $S$. For each cluster $j$ in layer $K$, there is a set of potential cluster heads in layer $K-1$, denoted as $H_{j} \subseteq S$. For cluster $j$ to select node $i \in H_{j}$, there is an associated cost $c_{j i}$ which is proportional to the energy consumption for data transmission from cluster $j$ to node $i$ and is adversely proportional to the residual energy of node $i$. Suppose the cluster pairs are determined based on the physical locations. For each pair of clusters $j$ and $k$, denoted as $\langle j, k\rangle$, let the common cluster heads be $H P_{j k}=H_{j} \cap H_{k}$. The problem is to select two cluster heads from $H P_{j k}$ for each pair of clusters $j$ and $k$ such that the total cost is minimized. The problem can be formulated as a classical transportation model of which the objective is to send flows with the minimum cost from a set of source nodes to a set of destination nodes satisfying capacity constraints on the source nodes and demand constraints on the destination nodes. A transportation problem can be readily solved by the network flow theory [1].

To have a transportation model for the dual cluster heads selection problem, we define a source node for each pair of clusters with the capacity of two units of flow, a destination node for each cluster head with one unit of flow demand. An arc is set from a source node to a destination node if 
the corresponding cluster head of the destination node is a possible choice for the corresponding cluster pair of the source node, and the unit flow cost of the arc is just the sum of cost from the two clusters in the pair to the cluster head. Fig. 5 shows the transport model for the dual cluster head selection problem.

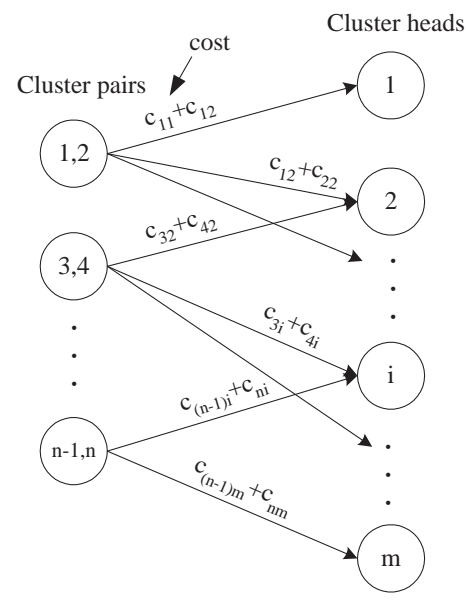

Figure 5. The transport model for the dual cluster head selection problem.

Specifically, we have the following formulation. For any cluster pair $\langle j, k\rangle$ and cluster head $i$ with $i \in H P_{j k}$, we define a variable $x_{j k i}$ to indicate the selection of cluster pair $<j, k>$ to cluster head $i$. Then we have

$$
\min \sum_{<j, k>} \sum_{i \in H P_{j k}}\left(c_{j i}+c_{k i}\right) x_{j k i}
$$

subject to

$$
\begin{gathered}
\sum_{i \in H P_{j k}} x_{j k i}=2, \quad \forall(j k) ; \\
\sum_{<j, k>} x_{j k i} \leq 1, \quad \forall i ; \\
x_{j k i}= \begin{cases}1 & \text { if cluster head } i \text { is selected by }<j, k>; \\
0 & \text { otherwise. }\end{cases}
\end{gathered}
$$

The sink node, which maintains the location and energy information of all the nodes, will run network flow algorithms to solve the formulated transportation problem for clusters at each layer in the network discovery phase. Then it allocates channels and decides the channel switching sequence for each cluster head. The channel allocation information will be updated in the $\mathrm{CP}$ of the data transmission phase.

\section{Performance Evaluation}

This section presents simulation results of the coordinated robust routing scheme on the layered architecture. A
C-language based discrete-event based simulator was developed. In the simulation, we assume a $250 m \times 250 m$ network with the sink node locates at the center and the number of nodes in the network $N \in\{200,400,600,800,1000\}$. The radio transmission range is set as 20 meters; the maximum buffer size is set to 500 packets per node; the number of cluster heads for each cluster is set to 2 ; the data frame size $S$ is set to 50 slots; and the initial energy per node is $10 \mathrm{~J}$. The transmission delay between each sensor to its cluster head is assumed at $2 \mathrm{~ms}$, and the processing delay (including time for data aggregation) of each sets of data is assumed at $100 \mu \mathrm{s}$.

We consider a simple energy consumption model as presented in $[5,8]$. The energy consumed by transmission $\left(E_{t}\right)$ and the energy consumed by receiving $\left(E_{r}\right)$ are calculated according to the following functions of the unit energy consumption of the transceiver $E_{e}$ and the unit energy consumption of the transmitter amplifier $E_{a}$.

$$
\begin{gathered}
E_{t}=E_{e} \times l+E_{a}(l, d) \\
E_{r}=E_{e} \times l
\end{gathered}
$$

where $l$ represents the packet length, $d$ represents the transmission range, and $E_{e}=50 \mathrm{~nJ} / \mathrm{bit}, E_{a}=100 \mathrm{pJ} / \mathrm{bit} / \mathrm{m}^{2}$.

We consider the following performance metrics vs. the number of nodes: the average packet latency in seconds, energy consumption per packet in Joules, and time to network partition. The energy consumption per packet is calculated over all the hops that a packet traverses. The time to network failure is defined as the time instance when the network is no longer connected due to node deaths. We compare the performance of the CRR scheme (in two formats: "dual head one buffer" and "dual head two buffer") with the single cluster head scheme.

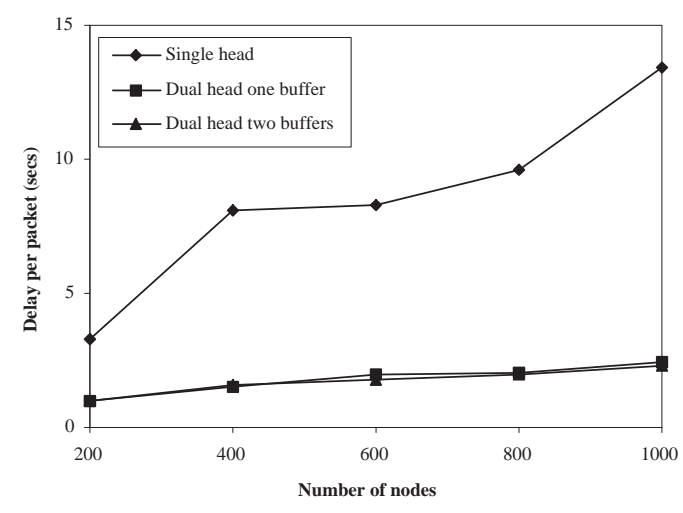

Figure 6 . The average packet delay vs. the number of nodes.

As shown in Fig. 6, the CRR scheme improves the average packet delay significantly compared with the single cluster head scheme. The "dual head two buffers" has less 
average delay than the "dual head one buffer". Fig. 7 shows the CRR scheme consumes less energy per packet than the single head scheme. Consistently, as shown in Fig. 8, the CRR scheme tends to have longer network life time than the single head scheme for different numbers of nodes. And the "dual head two buffers" achieves longer network life time than the "dual head one buffer".

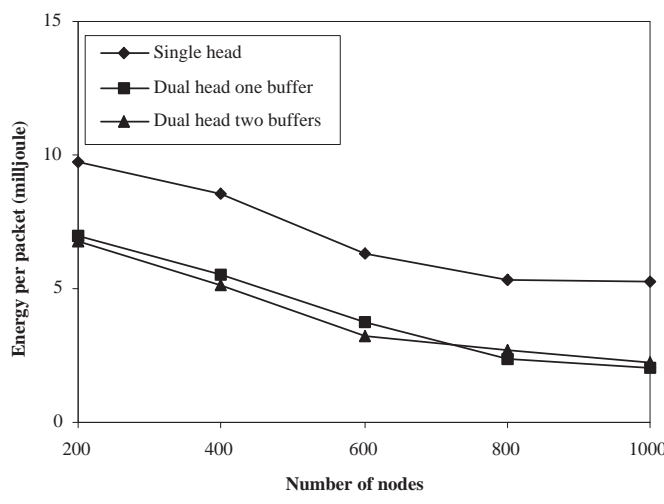

\section{Figure 7. The average energy per packet vs.} the number of nodes.

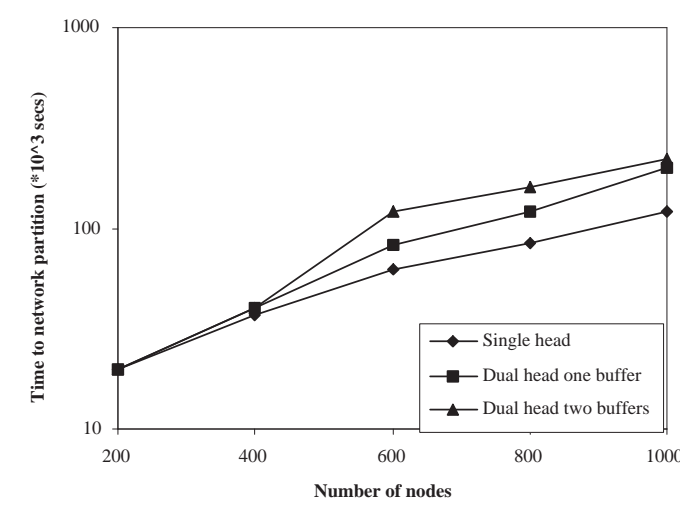

Figure 8. Time to network partition vs. the number of nodes.

\section{Summary}

In this paper, we consider the fault-tolerance problem for layered wireless sensor networks. We propose the coordinated robust routing scheme which constructs the robust routing trees coordinately from the most outward layer to the sink node. The coordination is achieved by selecting two dedicated cluster heads to every two clusters in one layer. By having two cluster heads for every cluster pair, the CRR scheme provides fault-tolerance and energy efficiency with reduced network redundancy. Through simulations, we show that the CRR scheme achieves less average packet delay, less energy consumption per packet, and longer network life time than the single cluster head scheme.

\section{References}

[1] R. K. Ahuja, T. L. Magnanti, and J. B. Orlin, Network Flows: Theory, Algorithms, and Applications, Prentice Hall, Upper Saddle River, New Jersey, 1993.

[2] I. F. Akyildiz, W. Su, Y. Sankarasubramaniam, and E. Cayirci, "A survey on sensor networks," IEEE Commu. Mag., pp. 102-114, Aug. 2002.

[3] E. H. Callaway, Wireless Sensor Networks: Architectures and Protocols, Auerbach Publications, 2004.

[4] A. P. Chandrakasan, R. Min, M. Bhardwaj, S. Cho, and A. Wang, "Power aware wireless microsensor systems," Keynote Paper in ESSCIRC, Floqrence, Italy, Sept. 2002.

[5] J. Ding, K. Sivalingam, R. Kashyapa, and L.J. Chuan, "A multi-layered architecture and protocols for largescale wireless sensor networks," in Proc. IEEE Semianual Vehicular Technology Conf., Oct. 2003.

[6] W. Heinzelman, "Application-specific protocol architectures for wireless networks," Ph. D. Thesis, Massachusetts Institute of Technology, June 2000.

[7] L. Krishnamachari, D. Estrin, and S. Wicker, "The impact of data aggregation in wireless sensor networks," in Proc. Int'l Conf. Distributed Computing Systems Workshops, 2002, pp. 575-578.

[8] S. Lindsey, K. M. Sivalingam, and C. S. Raghavendra, "Data gathering algorithms in sensor networks using energy metrics," IEEE Trans. Parallel and Distributed Systems, vol. 13, pp. 924-935, Sept. 2002.

[9] E. Shih, S. Cho, N. Ickes, R. Min, A. Sinha, A. Wang, and A. P. Chandrakasan, "Physical layer driven algorithm and protocol design for energy-efficient wireless sensor networks," in Proc. MOBICOM, 2001, pp. 272286.

[10] K. Sohrabi, J. Gao, V. Ailawadhi, and G. J. Pottie, "Protocols for self-organization of a wireless sensor network,” IEEE Pers. Commun., pp. 16-27, Oct. 2000.

[11] J. Wang, M. Yang, X. Qi, and R. Cook, "Dual-home based multicast protection," in Proc. GLOBECOM, 2004, pp. 1123-1127. 